\title{
ON THE REAL LINEAR POLARIZATION CONSTANT PROBLEM
}

\author{
Máté Matolcsi And Gustavo A. MuÑoz
}

Abstract. The present paper deals with lower bounds for the norm of products of linear forms. It has been proved by J. Arias-de-Reyna [2], that the so-called $n^{\text {th }}$ linear polarization constant $c_{n}\left(\mathbb{C}^{n}\right)$ is $n^{n / 2}$, for arbitrary $n \in \mathbb{N}$. The same value for $c_{n}\left(\mathbb{R}^{n}\right)$ is only conjectured. In a recent work A. Pappas and S. Révész prove that $c_{n}\left(\mathbb{R}^{n}\right)=n^{n / 2}$ for $n \leqslant 5$. Moreover, they show that if the linear forms are given as $f_{j}(x)=\left\langle x, a_{j}\right\rangle$, for some unit vectors $a_{j}(1 \leqslant j \leqslant n)$, then the product of the $f_{j}$ 's attains at least the value $n^{-n / 2}$ at the normalized signed sum of the vectors $\left\{a_{j}\right\}_{j=1}^{n}$ having maximal length. Thus they asked whether this phenomenon remains true for arbitrary $n \in \mathbb{N}$. We show that for vector systems $\left\{a_{j}\right\}_{j=1}^{n}$ close to an orthonormal system, the Pappas-Révész estimate does hold true. Furthermore, among these vector systems the only system giving $n^{-n / 2}$ as the norm of the product is the orthonormal system. On the other hand, for arbitrary vector systems we answer the question of A. Pappas and S. Révész in the negative when $n \in \mathbb{N}$ is large enough. We also discuss various further examples and counterexamples that may be instructive for further research towards the determination of $c_{n}\left(\mathbb{R}^{n}\right)$.

Mathematics subject classification (2000): 46G25, 52A40.

Key words and phrases: Linear polarizations constants, polynomials, polynomial norm estimates.

\section{REFERENCES}

[1] V. Anagnostopoulos, Sz. RÉVÉsZ, Polarization constants for products of linear functionals over $\mathbb{R}^{2}$ and $\mathbb{C}^{2}$ and Chebyshev constants of the unit sphere, Preprint of the Alfréd Rényi Mathematical Institute, Hung. Acad. Sci., 4, 2003, 9 pages.

[2] J. ARIAS-DE-REYNA, Gaussian Variables, polynomials and permanents, Linear algebra and its Applications 285, (1998), 395-408.

[3] K. M. BALL, The complex plank problem, Bull. London Math. Soc, 33, (2001), 433-442.

[4] K. M. BALL, The plank problem for symmetric bodies, Invent. Math., 104, (1991), 535-543.

[5] T. BANG, A solution of the plank problem, Proc. Amer. Math. Soc., 2, (1951), 990-993.

[6] C. BeníteZ, Y. SARANTOPOULOS AND A. M. TONGE, Lower bounds for norms of products of polynomials, Math. Proc. Camb. Phil. Soc., 124, (1998), 395-408.

[7] S. DINEEN, Complex analysis on infinite dimensional spaces, Springer Monographs in Mathematics, Springer-Verlag, Berlin, 1999.

[8] J. C. GARCíA-VÁZQUEZ, R. Villa, Lower bounds for multilinear forms defined on Hilbert spaces, Mathematika 46, (1999), 315-322.

[9] F. JoHN, Extremum problems with inequalities as subsidiary conditions, Courant Aniversary Volume, Interscience, New York, (1948), 187-204.

[10] A. E. LitvaK, V. D. Milman And G. Schechtman, Averages of norms and quasi-norms, Math. Ann. 312, (1998), 95-124.

[11] M. MARCus, The Hadamard theorem for permanents, Proc. Amer. Math. Soc., 15, (1964), 967-973.

[12] M. Marcus, H. Minc, The Pythegorean theorem in certain symmetric classes of tensors, Trans. Amer. Math. Soc., 104, (1962), 510-515.

[13] M. MatolCSI, Linear polarization constant of $\mathbb{R}^{n}$, Acta Math. Hung., Acta Math. Hung., 108, (1-2) (2005), 129-136. 
[14] M. MatolCsi, A geometric estimate on the norm of product of functionals, Preprint of the Alfréd Rényi Mathematical Institute, Hung. Acad. Sci., 2, (2004), 7 pages.

[15] G. A. Muñoz, Y. Sarantopoulos And A. M. Tonge, Complexifications of real Banach spaces, polynomials and multilinear maps, Studia Math., 134, (1999), 1-33.

[16] A. PAPPAS, Sz. RÉVÉSz, Linear polarization constants of Hilbert spaces, Preprint of the Alfréd Rényi Mathematical Institute, Hung. Acad. Sci., 3, (2003), 14 pages.

[17] Sz. Gy. RÉVÉSZ, Y. SARANTOPOULOS, Plank problems, polarization and Chebyshev constants, J. Korean Math. Soc., 41, (1) (2004), 157-174.

[18] R. RYan, B. TURetT, Geometry of Spaces of Polynomials, J. Math. Anal. Appl., 221, (1998), 698-711. 